\title{
Travelling Waves of Fast Cryo-chemical Transformations in Solids (Non-Arrhenius Chemistry of the Cold Universe)
}

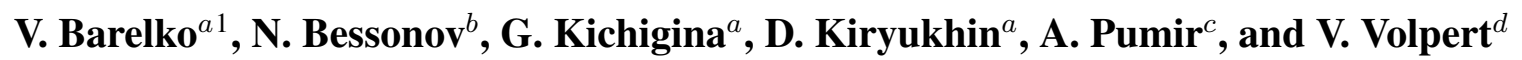 \\ ${ }^{a}$ Institute of Problems in Chemical Physics, 142432 Chernogolovka, Russia \\ ${ }^{b}$ Institute of Mechanical Engineering Problems, 199178 Saint Petersburg, Russia \\ ${ }^{c}$ Department of Mathematics, University of Nice, 06560, Valbonne, France \\ ${ }^{d}$ Institute of Mathematics, UMR 5208 CNRS, University Lyon 1, 69622 Villeurbanne, France
}

\begin{abstract}
Propagation of chemical waves at very low temperatures, observed experimentally [1] at velocities of order $10 \mathrm{~cm} / \mathrm{s}$, is due to a very non- standard physical mechanism. The energy liberated by the chemical reaction induces destruction of the material, thereby facilitating the reaction, a process very different from standard combustion. In this work we present recent experimental results and develop a new mathematical model which takes into account mechanical and chemical processes. We carry out numerical simulations and suggest a simplified model in order to obtain an explicit expression for the wave speed. This modelling allows us to give a possible explanation of the existence of two modes of propagation observed experimentally and of the dependence of the speed of propagation on the radiation dose.
\end{abstract}

Key words: cryo-chemical waves, experiments, modelling

AMS subject classification: $35 \mathrm{~K} 35,80 \mathrm{~A} 20$

\section{Introduction}

This work is devoted to a new field of applications of fracture mechanics in solid phase chemistry. A feedback coupling between fracture and activation of solid matrix can result in initiation of chemical reactions with non-Arrhenius kinetics. They can occur under the temperatures close to absolute zero. In particular, this can be one of possible mechanisms of fast chemical evolution in a solid state in the Universe [1], [5]. This mechanism can also be related to the formation of solid

\footnotetext{
${ }^{1}$ Corresponding author. E-mail: barelko@icp.ac.ru
} 
crust on the cold planets of the Solar system.

Autowave modes of fast chemical transformations caused by local brittle destruction of preradiolyzed solid samples were found when studying the mechanisms of chemical reactions at 4.2 - 77 K [1], [2]. Local brittle destruction of a long enough solid sample initiates chemical reactions on the freshly formed surface. They lead to temperature or density gradients which provide further layer-by-layer dispersion of a solid sample and the appearance of active surface on which chemical reactions develop. Due to positive feedback, chemical conversion propagates over the whole sample in the mode of a travelling wave front.

Such autowave modes of chemical conversion in solids with non-Arrhenius kinetics were observed for a number of chemical chain reactions including polymerization and co-polymerization at 4.2 - $77 \mathrm{~K}$ [7], [8]. The mechanism developed in [1], [2] suggests that an elastic energy accumulated in a frozen sample in conditions of radiolysis is liberated at the moment of brittle destruction resulting in the formation of chemically active molecules at the surface of cracks and in the increase of molecular mobility of the reagents. Thus, this specific "autowave tribochemical" mechanism can be important for low temperature chemical reactions where the conventional Arrhenius mechanism leads to effectively zero reaction rates.

Some effects of the elastic strain energy on post-radiation processes were reported in [3]-[12]. It was found that dynamics of autowave cryopolymerization of cyclopentadiene is essentially dependent on the conditions of wave initiation. For equal pre-radiation doses, the velocity of wave polymerization initiated by local brittle destruction at the bottom of the sample was found to be much higher than that initiated at the top of a solid sample. For the wave initiated from the bottom, the weight of the unreacted solid part of the sample increases the mechanical stress. This provides a more complete use of the energy accumulated in radiolysis at the moment of brittle destruction and accelerates the wave propagation [4]. Thus, if wave initiation of chemical conversion is realized by fracture from the top of the sample, then the mechanism of wave propagation is determined by heat transfer. If a wave is initiated from the bottom of the sample, the mechanism of propagation is different. It can be called a gasless detonation mechanism. Therefore, a top-initiated thermal wave is essentially slower than a detonation wave initiated from the bottom of the sample. It was shown for butyl chloride chlorination reaction that under certain initiation conditions the speed of the detonation wave differed by more than two orders of magnitude from the thermal wave [12].

In this paper we study cryo-chemical waves of acetaldehyde and $\mathrm{HCN}$ co-polymerization. This reaction is of a particular interest because it can provide a mechanism of formation of macromolecules in cosmic space. A solid-phase reaction in conditions of ultralow temperatures and radiation, particularly, acetaldehyde and $\mathrm{HCN}$ should be considered as a basis for prebiotic chemical evolution. These reactions provide a direct way of the formation of proteins and other large molecules in the Universe under the temperatures near absolute zero.

The first results on autowave solid-phase cryo-co-polymerization of acetaldehyde and HCN were presented in [11]. The work reported on reaction waves in a frozen $(77 \mathrm{~K})$ and pre-irradiated mixture in the thermal regime, i.e., the wave front was initiated from an open top of the sample. In contrast to the conventional linear dependence of the front velocity on radiation dose observed in other systems, polymerization of acetaldehyde and HCN demonstrated a fast saturation of the dependence of the wave velocity on pre-radiation dose and even formation of a maximum in this 
dependence. To elucidate the mechanism of co-polymerization of acetaldehyde and HCN we study a detonation-like wave initiated by local destruction of the sample in the closed bottom of an ampoule. A comparative analysis of the data presented below with the previous results [11] allows a possible explanation of the dependence of the wave velocities on radiation dose. This explanation is related to mechanic property of the solid matrix which becomes softer under large radiation does. It is confirmed experimentally.

The theory of cryo-chemical waves is based on the assumption that mechanical energy accumulated in the solid matrix can be transformed into chemical energy even at extremely low temperatures, therefore leading to rates of chemical transformation many orders of magnitude larger than predicted by the classical Arrhenius dependence. This energy transformation is a result of self-sustained brittle disruption of the solid matrix. This phenomenon can be called autowave self-sustained tribo-chemical mechanism.

The first simple theoretical model of autowave processes in solid-phase cryo-chemical reactions is proposed in [1], [5]. It is based on the assumption that brittle fracture is induced by thermal stress due to heat production because of the exothermal chemical reaction. This study is continued in [20] by the investigation of bifurcation phenomena and critical conditions with another mechanism of wave propagation. It takes into account the density change between the initial reagents and the products of reaction. This mechanism can provide a detonation-like propagation and wave velocities more than 1-2 orders of magnitude greater in comparison with the thermal propagation.

In the present paper we develop a more complete model of cryo-chemical waves which describes crack growth and its coupling with chemical reactions. We will carry out numerical simulations of the complete model and an analytical study of a simplified model. In the theoretical study, we will investigate the thermal regime propagation. However, we expect that the same model can also describe the detonation regime (see Discussion). This is a subject for future investigations.

The experiments show a non-monotone dependence of the speed of propagation on the radiation dose. It increases for small doses and decreases for large ones. Both observations can be explained in the framework of the model developed in this work by the variation of parameters characterizing the initial conditions or the properties of the solid matrix.

\section{Experiments}

In this section we study travelling waves of acetaldehyde and hydrogen cyanide cryocopolymerization. The effect of different initiation conditions on autowave co-polymerization of acetaldehyde and hydrogen cyanide will be analyzed. Local brittle destruction at the bottom of the sample provides a high-speed propagation of the reaction front. The velocity of the wave front is up to 1870 $\mathrm{mm} / \mathrm{s}$ for $55 \mathrm{kGy}$ ionizing radiation dose. However, the co-polymer formed in pre-irradiation conditions at $77 \mathrm{~K}$ plasticizes the solid matrix. As a consequence, degeneracy of high-speed mode (detonation-like) of co-polymerization wave is observed at high irradiation doses. Its velocity decreases to $50 \mathrm{~mm} / \mathrm{s}$ at $200 \mathrm{kGy}$ dose. Similarly, low propagation velocities are observed for the reactions initiated by brittle destruction at the top of the sample where the thermal mechanism of propagation is realized. 


\subsection{Experimental setup}

Hydrogen cyanide was prepared by decomposing potassium ferrocyanide with sulfuric acid according to the procedure described in [21]. The reaction product was additionally purified using low-temperature distillation (b.p. $=298.6 \mathrm{~K}, \mathrm{~m} . \mathrm{p} .=260 \mathrm{~K}$ ). Acetaldehyde was dried over calcium chloride and twice distilled in a rectifying column (b.p.=294 K).

Samples were radiolyzed at $77 \mathrm{~K}$ using a "Gammatok-100" setup with $0.5 \mathrm{~Gy} / \mathrm{s}$ Co gamma rays.

Autowave mode of co-polymerization was studied in 100-150 mm long cylindrical samples (5 $\mathrm{mm}$ in diameter) similar to those described in [1], [2]. Local brittle destruction of the samples (an initiation action) was realized using a constantan micro-heater frozen in the lower part of the sample at the bottom of the ampoule. Current pulsed through a micro-heater and stimulated local brittle destruction of the sample in the area adjacent to it. A co-polymerization wave propagating along the sample was registered with copper-constantan thermocouples located along the sample axis at a certain distance from each other.

Polymer conversion was determined gravimetrically after the removal of unreacted monomers in vacuum at room temperature.

The viscosity of the resulting co-polymers was measured at $293 \mathrm{~K}$. Since the co-polymer contained a small amount of $\mathrm{HCN}$ (0.4-4.0 mol. \%), its molecular mass (MM) was estimated from the equation: $[\eta]=5.36 \times 10^{-4} \mathrm{M}^{0.65}$ used for the determination of MM of polyacetaldehyde. Composition of the copolymers was calculated from the content of nitrogen determined by the elemental analysis.

\subsection{Results}

The study of the effect of initiation conditions on autowave co-polymerization of acetaldehyde and $\mathrm{HCN}$ was performed for a reagent mixture containing $25 \mathrm{~mol}$. \% of $\mathrm{HCN}$. Upon cooling down to $77 \mathrm{~K}$, the mixture transits to a glass-like state. A softening temperature (a transition from elastic to plastic state) is $90 \mathrm{~K}$, crystallization temperature is $110-115 \mathrm{~K}$ and melting point is $140 \mathrm{~K}$ (a detailed description of phase transformations at $77-200 \mathrm{~K}$ is presented for different initial ratios of co-monomers in [10]).

Peculiarities of travelling waves of acetaldehyde - HCN co-polymerization under initiating by local disturbance at the bottom of the sample should be compared with the experimental data reported in [11], where the travelling wave was initiated from the top. Local brittle destruction of the sample pre-radiolyzed at $77 \mathrm{~K}$ excites a travelling wave of co-polymerization of acetaldehyde and $\mathrm{HCN}$ in both initiation conditions (from the top or bottom of solid samples). The co-polymerization wave front propagates fast over the whole length of the sample (from the top to the bottom or from the bottom to the top) that is easily monitored on the sample because of color changes.

Figure 1 shows the dependence of the velocity of the co-polymerization waves on the preliminary $\gamma$-irradiation dose for the reaction initiated at the bottom (curve 2) and at the top (curve 1) of the sample. It was found that a critical preliminary $\gamma$-irradiation dose sufficient to initiate a travelling wave is somewhat lower $(10 \mathrm{kGy})$ when initiated from the bottom than from the top (15 $\mathrm{kGy}$ ), the propagation velocities being 6 and $18 \mathrm{~mm} / \mathrm{s}$, respectively. 


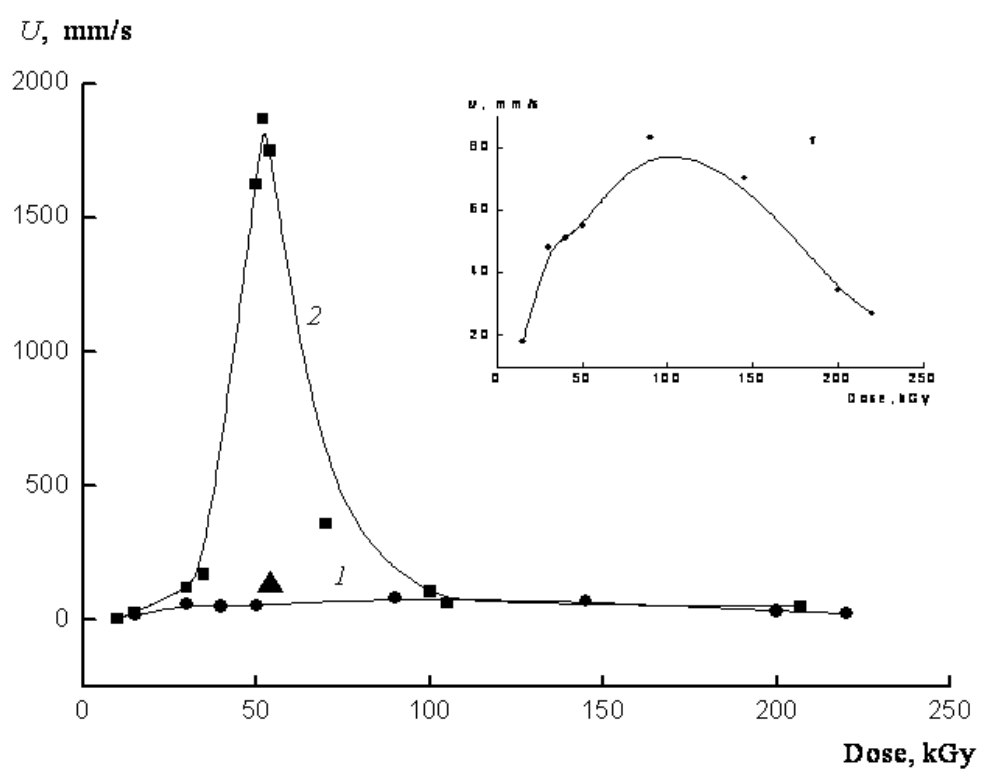

Figure 1: The dependence of reaction wave front propagation velocity on preliminary $\gamma$-radiation dose at $77 \mathrm{~K} .1$ - wave initiation at the top of the sample [4]; 2 - wave initiation at the bottom of the sample. Triangles - propagation velocity for the sample containing preliminarily added copolymer $(4 \%)$.

At 10-30 kGy radiation doses the values of front velocities are close for both initiation modes (from the top and from the bottom). However, an essential difference in propagation velocities is observed with further increase of the pre-radiation dose. If the reaction is initiated at the top of the sample, the co-polymerization front velocity monotonically increases to attain $80 \mathrm{~mm} / \mathrm{s}$ for $100 \mathrm{kGy}$ radiation dose and then decreases to $30 \mathrm{~mm} / \mathrm{s}$ at $220 \mathrm{kGy}$ dose (Figure 1, curve 1). If a conversion wave is initiated from the bottom of the sample (from the ampoule bottom), then at 40-70 kGy doses one observes a sharp increase of the wave speed. It attains $1900 \mathrm{~mm} / \mathrm{s}$ for 55 kGy dose and has a sharp maximum. Then a sharp decrease is observed, and at 100-220 kGy doses the wave speed is almost equal to that of the wave initiated from the top of the sample (Figure 1, curve 2).

Figure 2 shows typical temperature profiles vs. time registered using copper constantan thermocouples for different pre-radiation doses and for the reaction initiation at the top and at the bottom of the sample. First of all, common features of temperature profiles specific for both initiation modes should be noted. Similarly to other autowave processes [11], a co-polymerization reaction is switched on at the moment of local brittle destruction at $77 \mathrm{~K}$. The temperature in the reaction zone quickly attains its maximal value. It should be noted that the co-polymerization reaction does not stop immediately after the front passage. Further temperature registration shows a long temperature decrease with time. The characteristic time of this stage is several times longer 


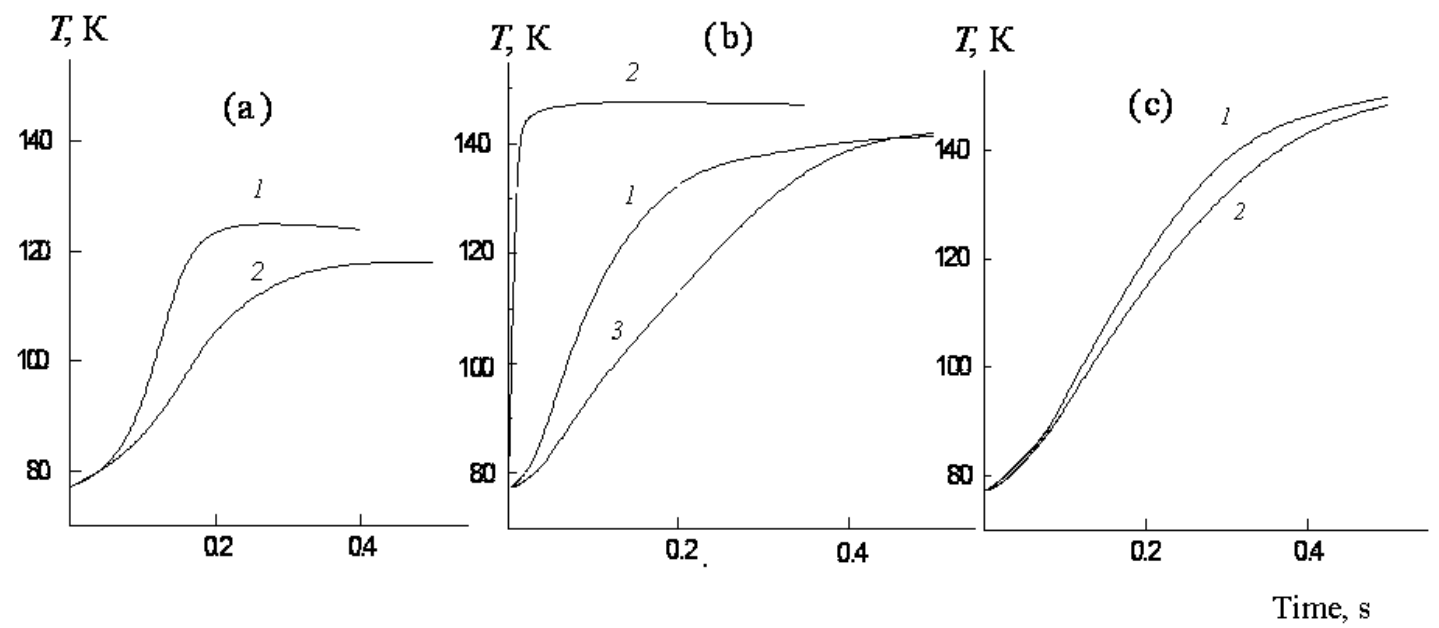

Figure 2: Time scanning for temperature profiles of autowave co-polymerization in the acetaldehyde+HCN system at $77 \mathrm{~K}$ for 15 (a), 50 (b), and 200 (c) kGy radiation dose. 1 - wave initiation in the top of the sample, 2, 3 - wave initiation in the bottom of the sample.

than the thermal inertia time of the sample. This shows that the reaction continues behind the front. The maximal temperature at the front increases from $113 \mathrm{~K}$ when initiated from the bottom (10 $\mathrm{kGy}$ radiation dose) and from $124 \mathrm{~K}$ when initiated from the top (15 kGy radiation dose) up to $140-150 \mathrm{~K}$ at $50-220 \mathrm{k}$ Gy radiation dose. The co-polymer yield increases proportionally to the maximal wave front temperature (Figure 3).

However, the shapes and dynamics of the temperature profiles are essentially dependent on the pre-radiation dose and reaction initiation conditions (from the top or from the bottom). At 10-30 $\mathrm{kGy}$ doses, the temperature profiles are close for both initiation modes: maximal temperature is attained after 0.2-0.4 s, the maximal temperature gradients are $15-30 \mathrm{~K} / \mathrm{mm}$ and the wave front width is within 3-8 mm (Figure 2a, curves 1 and 2). At 40-70 kGy radiation doses, the profiles are strongly different. The maximal temperature for the reaction initiated from the top of the sample is attained after $0.4-0.5 \mathrm{~s}$, whereas for the reaction initiated from the bottom after $0.02 \mathrm{~s}$ (Figure $2 \mathrm{~b}$, curves 1 and 2). At $>100 \mathrm{kGy}$ radiation doses, the temperature profiles become once again close for both initiation modes: maximal temperature is attained at 0.4-0.5 s, the maximal temperature gradients are $10-20 \mathrm{~K} / \mathrm{mm}$ and the reaction front width is within $8-15 \mathrm{~mm}$ (Figure 2c, curves 1 and 2).

When the wave propagation was finished, the samples were heated up to the room temperature and the co-polymer yield was determined. It is found that it increases with the pre-radiation dose to attain the maximal value of 45-50 \% (Figure 3). For comparison, in Figure 3 are also given the values of the co-polymer yield in travelling wave conditions for the reaction initiated from the top of the samples [11] and for the reaction in thermally activated post-effect conditions where, under slow heating the pre-irradiated acetaldehyde-HCN system, post-polymerization proceeds at 


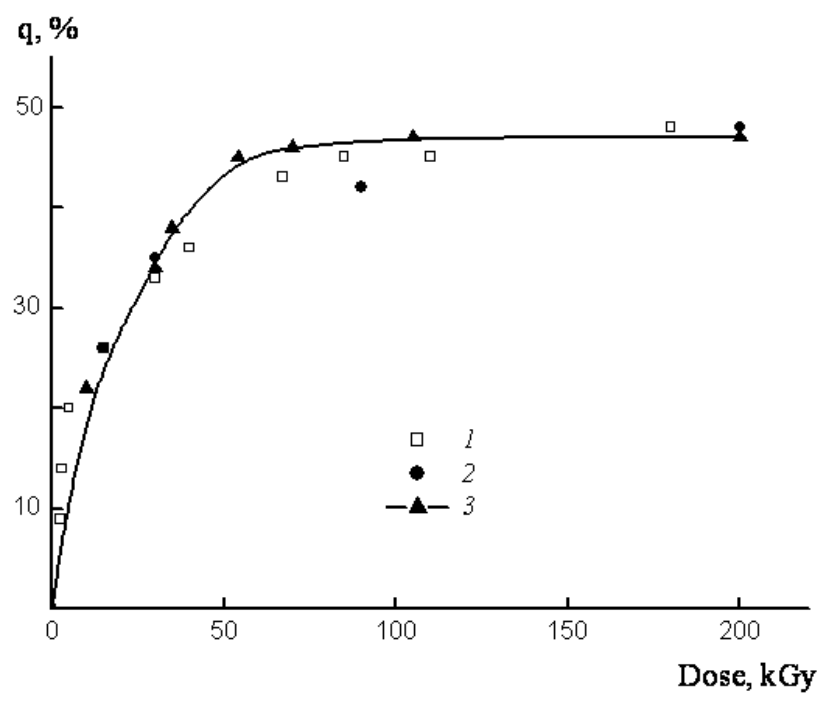

Figure 3: The dependence of the copolymer yield on the preliminary radiation dose at $77 \mathrm{~K} .1$ - thermally activated polymerization [11]; 2, 3 - autowave process initiated in the top and in the bottom of the sample, respectively.

the temperature close to that of softening state [10]). The dependence of the co-polymer yield on the radiation dose is almost similar for these different reaction conditions.

Figure 4 shows the dependence of the co-polymer molecular mass on the pre-irradiation dose for three conditions of reaction initiation: travelling wave conditions (top and bottom initiated) and thermally activated post-effect. The molecular mass of the co-polymer decreases with increasing the pre-irradiation dose almost similarly in all three cases. Somewhat lower molecular mass values can be noted only for the thermally activated post-effect as compared with propagating waves. This is possibly due to the fact that the wave front is initiated at lower temperatures $(77 \mathrm{~K})$ than the thermally activated post-polymerization (near softening temperatures, i.e. $T_{g}=90 \mathrm{~K}$ ): low temperatures provide a greater life-time of active polymerization centers.

The contents of nitrogen in the co-polymer for the waves initiated both from the top and from the bottom of the sample increases with the pre-radiation dose [11]. Calculations show that for 10-50 kGy pre-irradiation doses, the resulting macromolecules contain two HCN units. At 100$200 \mathrm{kGy}$ dose, a number of HCN units is approximately four. These data are close to those for thermally activated post-polymerization in the process of slow heating [11], [10]. 


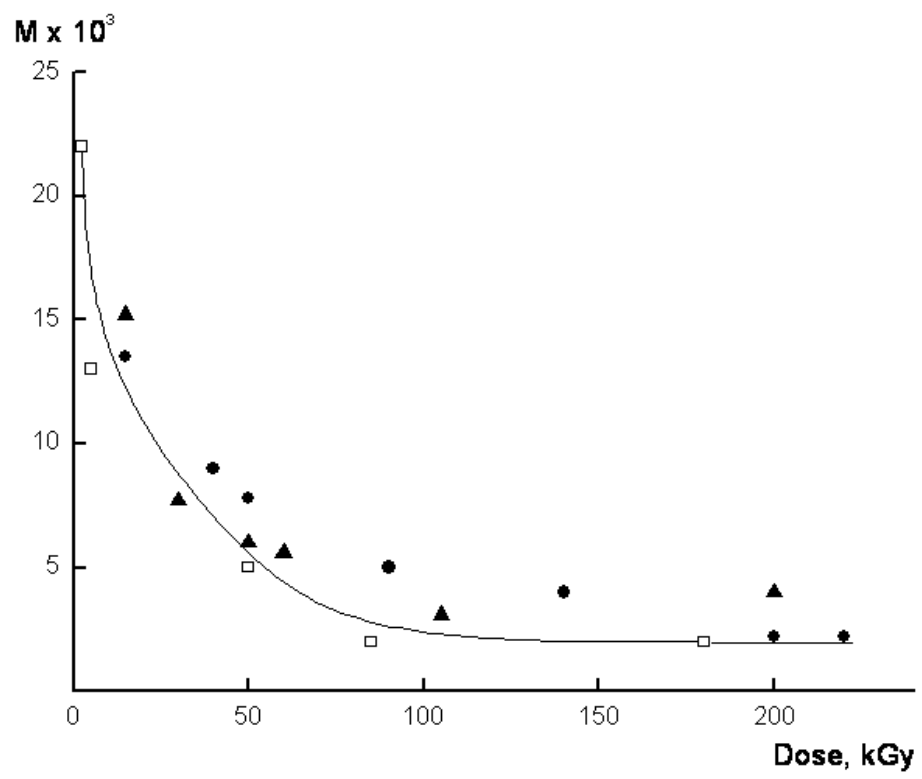

Figure 4: The dependence of the molecular mass on the radiation dose at $77 \mathrm{~K}$. Notations are the same as in Figure 3.

\section{Modelling}

\subsection{Governing equations}

As we discussed above, a new type of chemical waves was found experimentally in [1], [12]. It was observed that a reaction front propagating in a frozen sample with the temperature of the liquid nitrogen or the liquid helium was accompanied by destruction of the sample. In [1] the following physical mechanism was suggested. Heat release because of the reaction leads to appearance of temperature gradients in the sample and as a consequence to its destruction. The destruction of the sample leads, in turn, to liberation of energy. The active centers frozen in the solid matrix become movable, the reaction accelerates and results in a heat production, destruction of the next layer of the sample, and so on. The coupling between the chemical reaction and destruction of the sample leads to propagation of reaction fronts accompanied by a front of cracks. In this section we discuss a mathematical model of this process. Propagation of low-temperature waves is observed for different chemical systems [12]. So we can assume that the kinetics of the reaction is not very important for the front propagation. We consider here the one-step reaction of continuation of chain

$$
A+R \rightarrow B+R,
$$

where $A$ is the concentration of the reactant, $B$ the concentration of the product of the reaction, $R$ concentration of active centers. The system of equations describing distribution of the temperature, 
and of the concentrations has the form

$$
\begin{gathered}
\frac{\partial T}{\partial t}=\kappa \frac{\partial^{2} T}{\partial x^{2}}+q K(T) A R-\epsilon\left(T-T_{0}\right), \\
\frac{\partial A}{\partial t}=-K(T) A R \\
\frac{\partial R}{\partial t}=K_{1} \frac{\partial S}{\partial t}-K_{2} R .
\end{gathered}
$$

Here $T$ is the temperature, $\kappa$ the coefficient of thermal diffusivity, $q$ the adiabatic heat release, $\epsilon$ the coefficient of heat loss, $T_{0}$ the ambient temperature, $S$ the concentration of cracks, $\partial S / \partial t$ is the rate of crack growth, $K_{1}$ and $K_{2}$ are some positive parameters, which characterize the rate of appearance of active centers at the new surface and their death; $K(T)$ is the temperature dependence of the reaction rate. Taking into account that for cryo-chemical reactions the temperature dependence of the reaction rate is weak, we will assume that $K(T)$ is a constant.

In the equation (3.3) we assume that the rate of liberation of active centers is proportional to the rate of crack growth, i.e. to the rate of formation of the new surface. We consider the monomolecular death of active centers.

To complete the description of the model, we need to specify the rate of crack growth. It is known that temperature gradients decrease the critical force applied to the sample when cracks begin to grow [22]. Assuming that the characteristic dimension of cracks is essentially less than the preheat zone of the reaction front and that the distance between cracks is sufficiently large, we can neglect their mutual influence and suppose that the cracks in the preheat zone are in a homogeneous heat flow. Then the critical stress and the starting speed of crack growth can be written in the form:

$$
\begin{gathered}
p_{c}=c_{1} \frac{k_{c}}{\sqrt{s}}-c_{2} \beta E_{0} s Q, \\
v=\frac{v_{0}}{\sqrt{2(1+\nu)}} \sqrt{1-\left(\frac{p_{c}}{p}\right)^{2}}, p \geq p_{c}
\end{gathered}
$$

and $v=0$ for $p<p_{c}$. Here $s$ is the length of the crack, $k_{c}$ is the viscosity of destruction, $E_{0}$ the Young modulus, $Q$ the heat flux, $v_{0}$ the speed of elastic waves, $\nu$ the Poisson coefficient, $p$ the stress in the sample, $\beta$ the coefficient of thermal expansion, $c_{1}$ and $c_{2}$ numerical factors.

The stresses appear in the sample in the process of its preparation [1] when a tube with the liquid reactants is frozen by the liquid nitrogen or by the liquid helium. We consider an average value $p$ of the stresses in the sample as a given parameter.

Cracks can develop from microdefects which are always present in the sample. We assume that the defects and the cracks are sufficiently small and do not interact with each other. If initially cracks are not exactly the same and can have different lengths, then we need to introduce their distribution with respect to length. Let $\phi(s)$ be the density of distribution, and $H(s, Q)$ be the speed of growth of the crack as a function of its length and of the heat flux, $H(s, Q)=v$, where $v$ is given by (3.5). This distribution will change due to length growth. It is described by the transport equation

$$
\frac{\partial \phi}{\partial t}+\frac{\partial(H \phi)}{\partial s}=0
$$


The total rate of crack growth, which should be substituted in (3.3), is

$$
\frac{\partial S}{\partial t}=\int_{0}^{\infty} \phi(s, t) H(s, Q) d s
$$

System (3.1)-(3.6) should be completed by initial and boundary conditions. They will be given in the next section for the dimensionless model.

\subsection{Dimensionless model}

We introduce dimensionless variables scaling time by $K$, the space variable by $\sqrt{K / \kappa}$, the concentration $A$ by its maximal value at infinity. The dimensionless temperature is given by the expression $\theta=\left(T-T_{0}\right) / q$. In terms of the scaled variables, problem (3.1)-(3.6), where we keep for convenience some of the notations, becomes:

$$
\begin{gathered}
\frac{\partial \theta}{\partial t}=\frac{\partial^{2} \theta}{\partial x^{2}}+A R-\sigma_{1} \theta \\
\frac{\partial A}{\partial t}=-A R \\
\frac{\partial R}{\partial t}=W-\sigma_{2} R \\
\frac{\partial \phi}{\partial t}+\frac{\partial(H \phi)}{\partial s}=0
\end{gathered}
$$

in the domain $0 \leq x \leq L, s_{0} \leq s \leq s_{1}$ with the boundary conditions

$$
\begin{gathered}
x=0: \frac{\partial \theta}{\partial x}=0, \quad x=L: \theta=0 \\
s=s_{0}: \phi=\phi_{0}
\end{gathered}
$$

and initial conditions

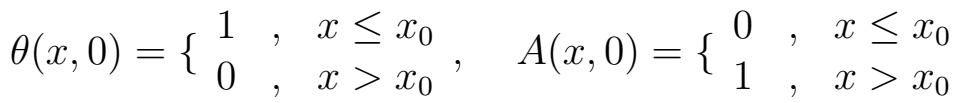

$$
\begin{aligned}
& R=0, \quad 0 \leq x \leq L, \quad \phi(x, s, 0)=\phi^{0}(s),
\end{aligned}
$$

where $\sigma_{1}$ and $\sigma_{2}$ are the coefficients of heat loss and of the loss of active centers,

$$
\phi^{0}(s)=\phi_{0} e^{-p(s-q)^{2}} / e^{-p\left(s_{0}-q\right)^{2}},
$$

and $q \in\left(s_{0}, s_{1}\right), p$ is a positive number. Furthermore, 


$$
\begin{aligned}
& W=\int_{s_{0}}^{s_{1}} \phi(x, s, t) H\left(s, \frac{\partial \theta}{\partial x}\right) d s \\
& H=\left\{\begin{array}{cc}
c \sqrt{1-F^{2}} & , \quad F \leq 1 \\
0 & , \quad F>1
\end{array}\right. \\
& F=\left\{\begin{array}{ccc}
\frac{a}{\sqrt{s}}-b s\left|\frac{\partial \theta}{\partial x}\right| & , & \text { if this expression }>0 \\
0 & , & \text { otherwise }
\end{array}\right.
\end{aligned}
$$

The values of positive parameters $a, b$, and $c$ are difficult to determine from the experimental data. They will be varied in numerical simulations.

\subsection{Numerical simulations}

\subsubsection{Structure of the wave}

Problem (3.8)-(3.14) can describe propagation of travelling waves. The evolution of the temperature distribution in time is shown in Figure 5. It has a specific for travelling waves form: the temperature profile remains the same and moves to the right with a constant speed.

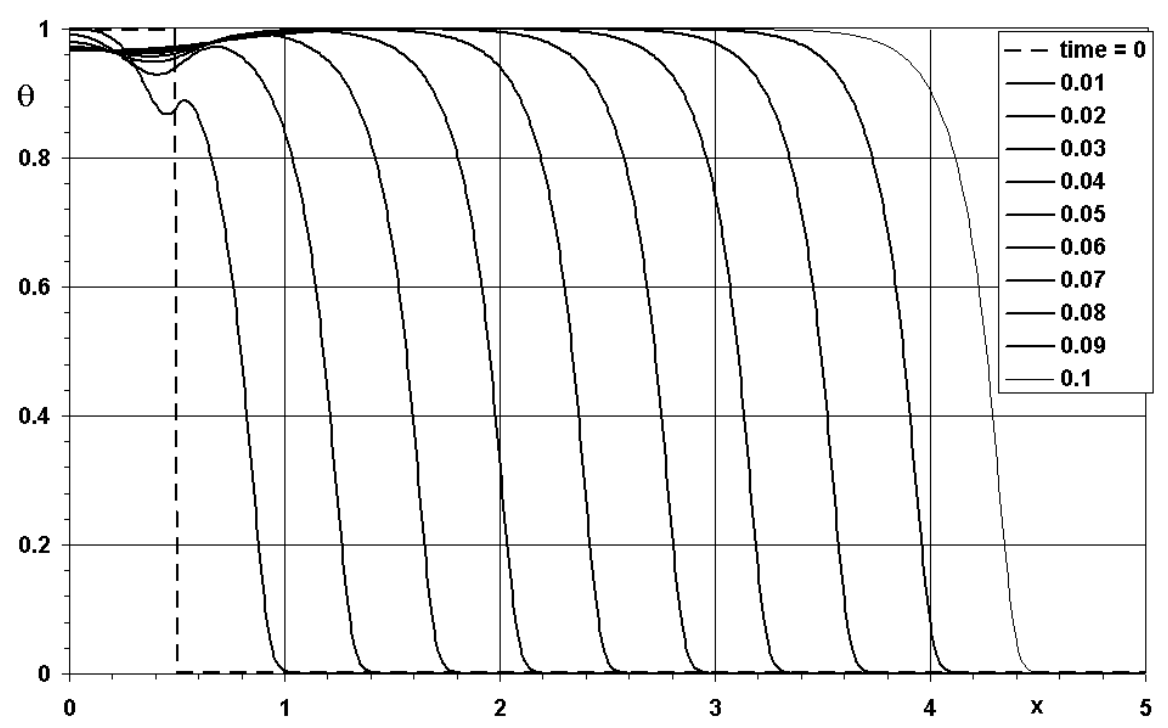

Figure 5: Temperature distribution in consecutive moments of time

The structure of the wave is shown in Figure 6 for the values of parameters $a=1, b=50, c=$ $50, s_{0}=0.01, s_{1}=0.2, p=4000, q=0.05, \phi_{0}=100, \sigma_{1}=\sigma_{2}=0$. The wave propagates from the left to the right. The blue curve in Figure 5 a) shows the temperature distribution, the green curve is the concentration $A$, and the red curve shows $R$. 


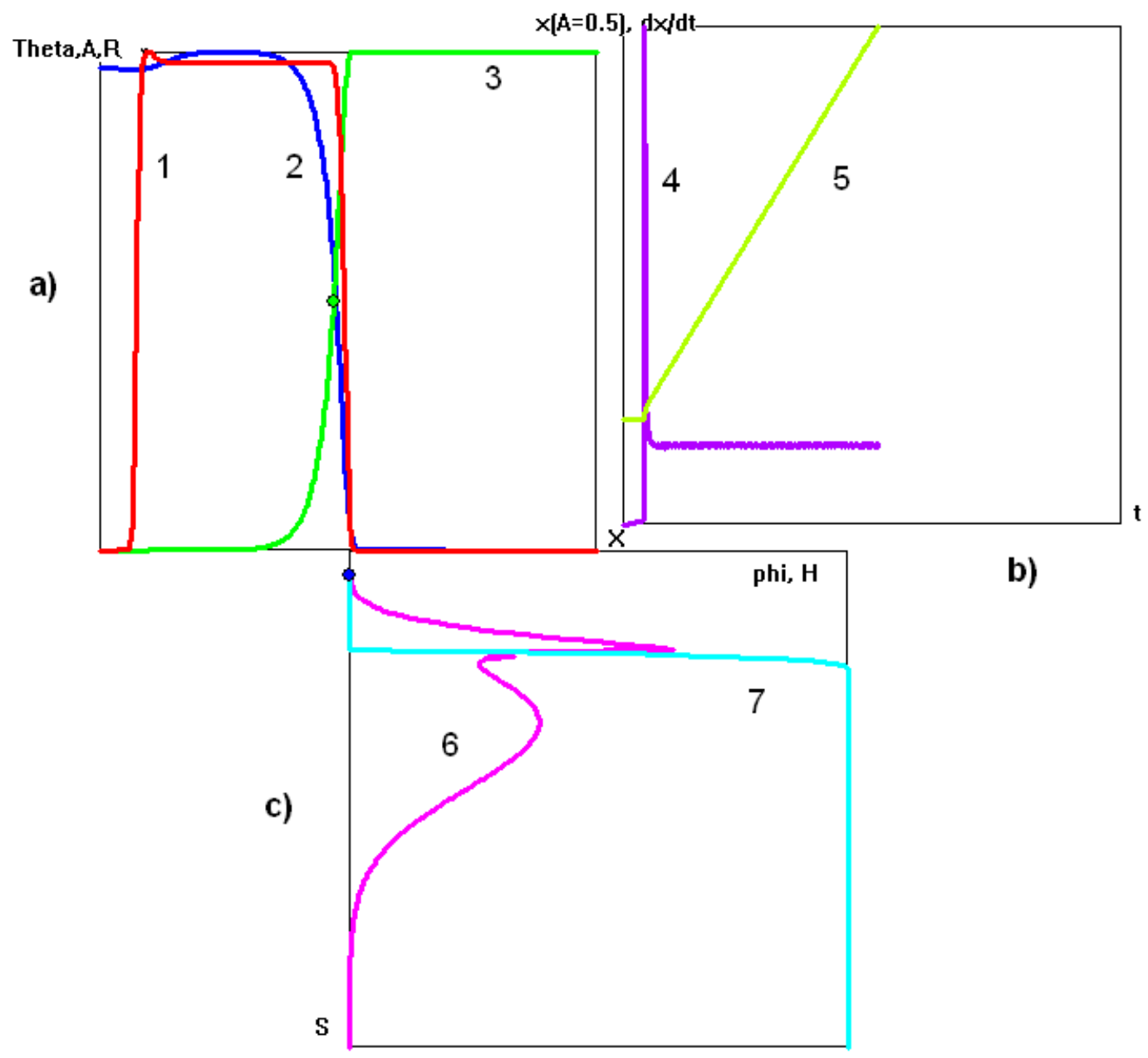

Figure 6: Wave structure, a) distributions of $R$ (curve 1), $\theta$ (curve 2), and $A$ (curve 3 ) for a fixed time, b) speed (curve 4) and front location (curve 5) as a function of time, c) distributions of cracks (curve 6) and the speed of their growth (curve 7) at a fixed space point.

When the temperature gradient at a given space point $x$ reaches some critical value, cracks start growing resulting in production of active centers $R$. Their concentration quickly reaches their maximum value $R_{m}$, which remains constant behind the front if $\sigma_{2}=0$.

The space interval where $R$ grows is strongly localized. The reaction zone, that is the space interval where $A$ is consumed is wider. It is basically located in the region where $R=R_{m}$. The temperature variation occurs in approximately the same region. The dimensionless temperature behind the reaction zone is approximately 1 and $A \approx 0$.

After a short transition period, the wave speed reaches its steady value, $c=38$ (Figure $6 \mathrm{~b}$ ), violate curve). The green curve in Figure $6 \mathrm{~b}$ ) shows the front location in time, determined as a coordinate of the point where $A=0.5$.

Figure $6 \mathrm{c}$ ) shows the evolution of cracks at the middle of the interval. The red line describes 
their distribution density with respect to the length, the blue line shows the speed of their growth. In the region where it is positive, the cracks grow, and the corresponding part of their length distribution moves in the direction of larger $s$. For the values of parameters under consideration, after some time they reach their maximum length $s=0.2$.

\subsubsection{Dependence on parameters}

There is a relatively small number of parameters in the dimensionless model. The constants $a, b$, and $c$ characterize mechanical properties of the material related to its destruction, $\phi_{0}, p, q, s_{0}, s_{1}$ determine the distribution of cracks with respect to length. We note that there is no parameter in the dimensionless model which would explicitly characterize the intensity of irradiation during the preparation of the sample. However, it has an important role because it determines the concentration of active centers frozen in the solid matrix and, as a consequence, the intensity of chemical reaction after the destruction of the sample. It can be easily verify that such a parameter enters as a numerical factor at the rate of production of active centers $W$. Therefore, it enters in the same way as the parameter $\phi_{0}$, which determines the number of micro-defects in the sample. Thus, we will vary $\phi_{0}$ taking into account that this is equivalent to the variation of intensity of irradiation.

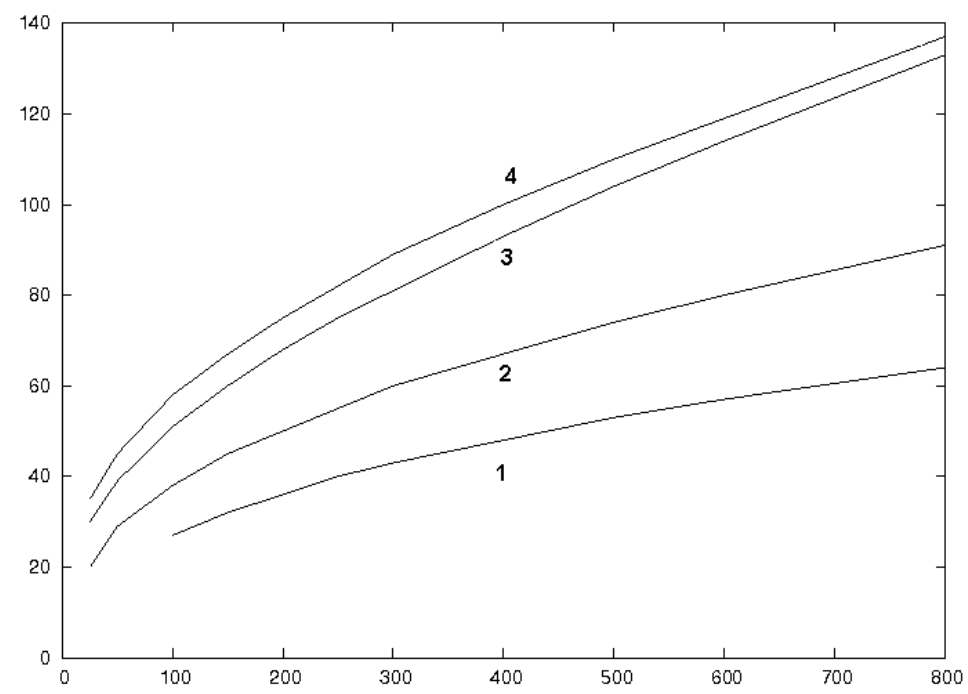

Figure 7: Wave speed as a function of $\phi_{0}$ for different values of b: 1) 25, 2) 50, 3) 100, 4) 150 .

Figure 7 shows the dependence of the speed of propagation on $\phi_{0}$ for different values of $b$. As it can be expected, the speed increases with the increase of $\phi_{0}$ because this corresponds to the increase of the number of active centers and of the reaction rate. For large values of $\phi_{0}$ this dependence becomes close to a linear function. We will discuss these results below in relation with the experiments. 

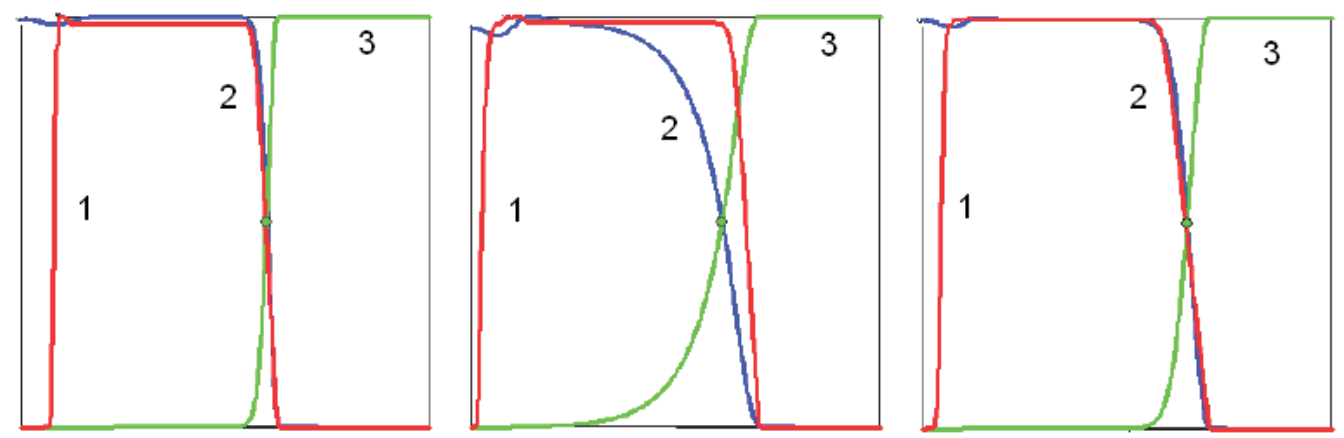

Figure 8: Active center (curve 1), temperature (curve 2), and concentration (curve 3) distributions for $\phi_{0}=800, b=50$ (left), $\phi_{0}=100, b=500$ (center), $\phi_{0}=800, b=500$ (right) and all other parameters as in Figure 5.

The increase of $b$ signifies that the reaction begins for smaller values of the temperature gradient. As a result, the speed of propagation increases. For small $b$, the dependence on this parameter is rather strong. For large values, it becomes weaker and approximately linear (not shown).

The variation of $\phi_{0}$ and $b$ preserves the qualitative structure of the wave. Figure 8 shows the distribution of the temperature and of the concentrations for three sets of parameters. It is interesting to note that for $\phi_{0}$ large, the profiles of $\theta$ and $R$ practically coincide. If $b$ is large, the temperature distribution becomes wider.

We note that the numerical method becomes unstable for small values of $\phi_{0}(<30)$ and for large values $(>1000)$. The wave speed begins to oscillate which makes it necessary to strongly decrease the time step.

Up to now, we have discussed propagation of waves without heat loss and without loss of active centers, that is $\sigma_{1}=\sigma_{2}=0$. Figure 8 (left) shows the temperature and concentration distributions for $\sigma_{1}=\sigma_{2}=80$. The color code here is the same as in Figure 6 a). The temperature and the concentration of $R$ drop down behind the front. When the concentration of $R$ becomes sufficiently low, the reaction stops resulting in the incomplete conversion: the concentration of $A$ remains positive behind the front. The dependence of the final depth of conversion behind the front on $\phi_{0}$ is shown in Figure 9 (right) (cf. Figure 3).

\subsection{Approximate solution}

Travelling wave solution of system (3.8)-(3.11) satisfies the system of equations

$$
\begin{gathered}
\theta^{\prime \prime}+c \theta^{\prime}+A R=0, \\
c A^{\prime}-A R=0, \\
c R^{\prime}+W=0,
\end{gathered}
$$



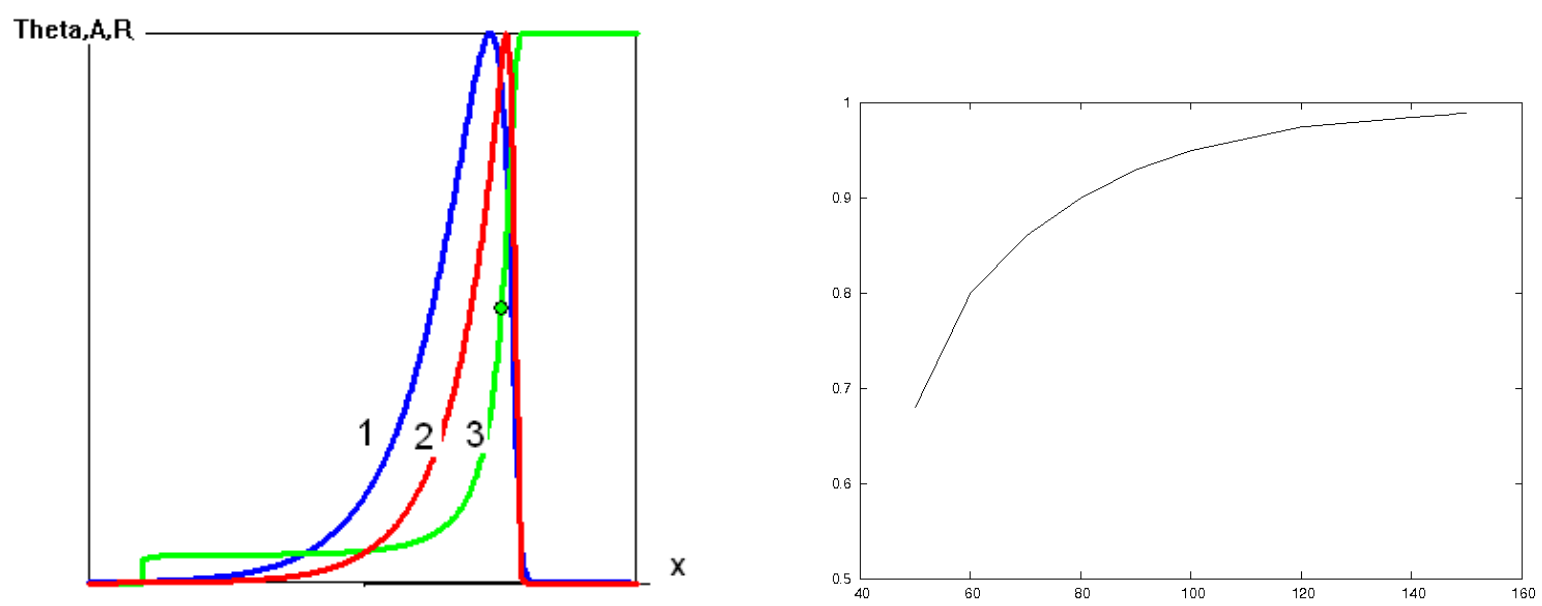

Figure 9: Wave propagation with heat loss and loss of active centers. Left: temperature (curve 1), active center (curve 2), and concentration (curve 3) distributions; right: final depth of conversion $(1-A)$ as a function of $\phi_{0}$.

Here prime denotes the derivative with respect to $x, c$ denotes the wave speed, and we assume that $\sigma_{1}=\sigma_{2}=0$. We look for a solution of this system for all $x$ with the limits at infinity:

$$
\theta(-\infty)=1, \theta(+\infty)=0, \quad A(-\infty)=0, A(+\infty)=1
$$

The term $W$ in equation (3.17) is given by expression (3.12). The rate of crack growth $H$ becomes constant if $F<0$ (Figure $10 \mathrm{~b}$ ). The integral of $\phi$ with respect to $s$, that is the total number of cracks, is independent of $x$ if we do not take into account the incoming and outgoing fluxes at the boundary of the interval $\left[s_{0}, s_{1}\right]$ (Figure 10 a). Therefore, it can be justified to approximate the rate $W$ of production of $R$ by a piece-wise constant function:

$$
W=\left\{\begin{array}{cc}
\omega, \quad-\tau \leq x \leq 0 \\
0, \quad \text { otherwise }
\end{array} .\right.
$$

Then from equation (3.17) we obtain

$$
R=\left\{\begin{array}{ccc}
0 & , & x \geq 0 \\
-R_{m} x / \tau & , & -\tau \leq x \leq 0 \\
R_{m} & , & x \leq-\tau
\end{array}\right.
$$

where $R_{m}=\omega \tau / c$. Equations (3.16) and (3.15) allow us to find first $A$ and then $\theta$ :

$$
A=\left\{\begin{array}{c}
1 \\
\exp \left(-r x^{2} /(2 \tau)\right) \\
\exp (r(x+\tau / 2))
\end{array} \quad, \quad \theta=\left\{\begin{array}{cc}
\theta_{0} \exp (-c x) & x \geq 0 \\
\exp (-c x)\left(\theta_{0}-\int_{0}^{x} \exp (c y) f(y) d y\right) & , \quad-\tau \leq x \leq 0 \\
1-c \exp (r(x+\tau / 2)) /(r+c) & , \quad x \leq-\tau
\end{array}\right.\right.
$$


where $r=R_{m} / c$ and $\theta_{0}=\theta(0)$,

$$
f(x)=c\left(1-e^{-r x^{2} /(2 \tau)}\right)
$$

The conditions that these functions are continuous together with their first derivatives allow us to find $\theta_{0}$ from the expression

$$
1-\frac{c}{r+c} e^{-r \tau / 2}=e^{c \tau}\left(\theta_{0}-\int_{-\tau}^{0} e^{c y} f(y) d y\right)
$$

The form of $W$ implies that the reaction begins at $x=0$, that is $\left|\theta^{\prime}(0)\right|=\Gamma$, where $\Gamma$ is the critical value of the temperature gradient for which $F=1$. Therefore

$$
\Gamma=\frac{a}{b s^{3 / 2}}-\frac{1}{b s}
$$

Here $s$ is the length of the crack which begins to grow. Since $s_{0} \leq s \leq s_{1}$, the we can estimate it by the limiting values or take the point of the maximum of the density distribution $\phi(s)$.
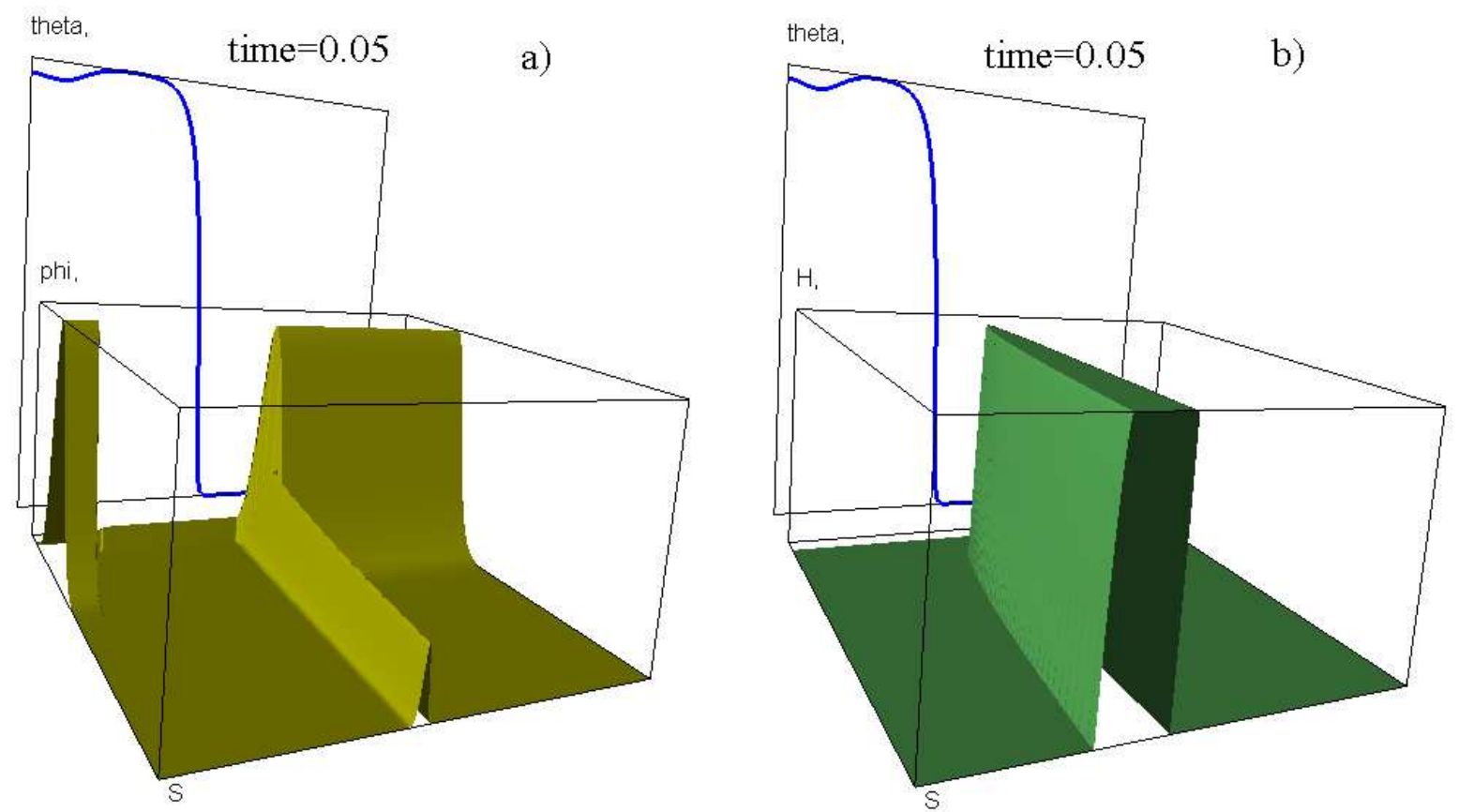

Figure 10: Temperature distribution (blue line), and the functions $\phi(x, s)(\mathrm{a}), H(x, s)(\mathrm{b})$.

Since $\theta^{\prime}(0)=-c \theta(0)$, we obtain the relation

$$
\Gamma=c \theta_{0}
$$


Together with (3.18), it gives us the equation with respect to $c$. Since it is complex and difficult to analyze, we consider a simpler limiting case where $\tau=0$. This means that the function $R$ becomes discontinuous. We obtain the formula for $A$ and $\theta$ :

$$
A=\left\{\begin{array}{c}
1 \\
\exp (r x)
\end{array}, \quad \theta=\left\{\begin{array}{cl}
r \exp (-c x) /(r+c) & , \quad x \geq 0 \\
1-c \exp (r x) /(r+c) & , \quad x \leq 0
\end{array},\right.\right.
$$

and the equation for the wave speed:

$$
R_{m} c=\Gamma\left(R_{m}+c^{2}\right) .
$$

Therefore, we obtain

$$
c_{ \pm}=\frac{R_{m}}{2 \Gamma} \pm \sqrt{\left(\frac{R_{m}}{2 \Gamma}\right)^{2}-R_{m}} .
$$

Hence, the condition of wave existence is given by the inequality

$$
R_{m} \geq 4 \Gamma^{2}
$$

This condition means that the critical temperature gradient should be sufficiently small or the concentration of active centers sufficiently high. Both of them has a clear physical meaning. If condition (3.21) is satisfied, then there are two waves with the speeds given by (3.20). In order to determine which one of them is physically realistic, we note that when $\Gamma$ decreases, the value $c_{-}$ also decreases, while $c_{+}$increases. Hence, we should use the larger value of the wave speed.

Formula (3.20) gives a reasonable approximation of the wave speed found numerically for large $b$, small $\phi_{0}$ and narrow distributions $\phi(s)$. For example, for the values of parameters $a=1, b=$ $400, c=50, p=8000, \phi_{0}=0.02, q=0.05, s_{0}=0.01, s_{1}=0.2$, we have from the numerical simulations $R_{m}=22, c=35$. If we use the maximum $s=p$ of the distribution $\phi(s)$ in order to compute $\Gamma$, we obtain $\Gamma \approx 0.7$. Then $c_{+} \approx 31$. However, for other values of parameters, the difference between numerical and analytical values of the wave speed can be essential. Analytical approximation of the speed requires further investigation.

\section{Discussion}

Fast cryo-chemical reactions represent a new mechanism of chemical reactions under the temperatures close to absolute zero. They can possibly occur in frozen mixtures of compounds such as ammonia and methane that are found in appreciable amounts in crusts of the cold planets of the Solar system.

In the experimental part of this work, we study cryo-chemical waves, with both thermal and detonation mechanisms. This part gives information about degeneration of detonation waves in solid matrixes and reasons of the phenomenon for example of copolymerization of acetaldehyde and HCN (the substances are typically ones for cosmic space).

In this paper, we have introduced a new mathematical model of cryo-chemical wave propagation, based on a coupling between the propagation of cracks and the chemical reaction. A more 
refined description of the dynamics of the fracture, a crucial aspect of this work, has been used here (cf. [18] - [20]). While the results obtained here generally confirm the picture obtained with the help of simpler models, it allows us to address more realistically a number of questions.

The model developed in this work allows us to give a possible explanation of the existence of two regimes of propagation, thermal regime and detonation, and of the non-monotone dependence of the speed of propagation on the radiation dose.

\subsection{Different modes of propagation}

\subsubsection{Thermal mechanism of propagation}

The before developed theoretical model was based on the idea of a key role of sharp temperature gradients appearing in a travelling wave front [1], [2]. Such gradients provoked the destruction and dispersion of a reagent layer adjacent to the front, and namely this factor is a reason for the excitation of chemical reaction in solid state. This mechanical-thermal mechanism was considered as a basic concept of a positive feedback coupling between an exothermal reaction and the destruction of a reacting solid matrix, which provided conditions for the formation of self-sustaining layer-by-layer (autowave) transformation of reagents.

This physical mechanism can be described by different mathematical models. In the previous works [18] - [20], the authors developed a model based on a heat equation with the source term depending of the temperature gradient. In this work, we suggest a more complete model which includes heat transfer, destruction of the sample and production of active centers. The simplified model used for the analytical study is close to the previous models in the sense that the source terms is a piece-wise constant function which becomes nonzero when the temperature gradient reaches a critical value. The difference of the simplified model suggested in this work is that it contains not only the heat equation but also the equation for the reactant.

\subsubsection{Detonation}

One of the features of autowave co-polymerization of acetaldehyde and $\mathrm{HCN}$ initiated by local brittle destruction from bottom of a sample is a sharp increase in velocities of travelling waves in comparison with the wave initiated from the top [4], [12]. The difference in velocities can be two orders of magnitude.

In order to explain this effect, a mechanism based on changes in density of reacting solid matter was suggested [19]. This factor can be one more physical reason for the formation of stress field in a wave front breaking a solid matrix. Fast local density change in a solid-phase conversion zone can be a source of a shock action on a neighboring area of a sample. The shock can break up a portion of a matrix and initiate a reaction in a local layer. This scheme of layer-by-layer shock acts provides conditions for the formation of a self-sustaining high-velocity travelling wave of solid matrix transformation realized due to non-thermal shock-wave (detonation-like) mechanism of chemical transformation in solid phases at ultralow temperatures.

Such detonation-like autowave process was realized for chlorination of butyl chloride when the reaction was initiated from the bottom of the ampoule [12]. It was found that at 3.0-4.0 kGy 
irradiation doses one observed a more than an order of magnitude increase in conversion front propagation velocity, namely, from 8 (for case of mechanical-thermal mechanism) up to $100 \mathrm{~cm} / \mathrm{s}$. The wave propagation velocity increased with pre-radiation dose and attained $800 \mathrm{~cm} / \mathrm{s}$ at $100 \mathrm{kGy}$. We suppose that a similar shock-wave mechanism is realized in the case of co-polymerization of acetaldehyde and $\mathrm{HCN}$ in conditions of wave initiation from bottom of the sample.

The model developed in this work does not take into account the change of medium density and its possible relation with the destruction of the sample. Moreover, the length of cracks is supposed to be much less than the zone of temperature variation. In fact, the cracks in the model are mathematical points. From the physical point of view this means that they are much shorter than the characteristic temperature scale or that they propagate in the direction perpendicular to the direction of the travelling wave.

If we take into account the final length of cracks and allow them to propagate in the same direction as the wave, then the mechanism of propagation can change. Indeed, if the crack grows forward faster than the thermal wave, then it will initiate chemical reaction in the region where it is not yet started. In this case the speed of propagation is determined by the speed of crack growth and it can attain the sound velocity.

It remains to explain why high-speed waves are observed under the initiation from the bottom and not from the top. The direction of propagation can play a role only if we take gravity into account. If the stress in the sample is determined by its weight, then cracks will propagate upwards. In the case of the front propagating also upwards, they can accelerate it by the mechanism described above. If the front propagates downwards, cracks will propagate in the direction of the reacted medium and will not increase the speed of front propagation.

\subsection{Numerical and analytical results}

The complete model is studied numerically. In order to obtain analytical results, we suggest a simplified model where the concentration of active centers $R$ is a piece-wise constant function: it is zero for $x>x_{0}$ where $x_{0}$ is the space point where the temperature gradient equals the critical value. It equals some positive constant behind $x_{0}$.

This approximation is in agreement with the results of numerical simulations where the function $R(x)$ is close to a piece-wise constant function if there is no loss of active centers $\left(\sigma_{2}=0\right)$. The value $R_{m}$ behind the front depends on the function $\phi^{0}(s)$, that is the initial distribution of defects. It is practically independent of the parameters $a, b$, and $c$ in a wide range of their variation.

The analytical formula for the wave velocity gives a reasonable approximation of the speed found numerically. It suggests a linear dependence of the speed on $R$ and on $b$ when these values are sufficiently large. The same behavior is observed in numerical simulations.

\subsection{Speed dependence on the radiation dose}

The theoretical results describe the increase of the wave speed with the increase of the radiation dose observed experimentally. However, it is not clear why travelling wave velocity decreases after achieving the maximum when then the dose attained $55 \mathrm{kGy}$ and then (at 100-220 kGy) the wave 
propagation velocity decreases to the value corresponding to that characteristic of the reaction initiated from top of the sample (Figure 1, curves 1 and 2).

We suggest that the decrease of reaction wave velocities is due to the formation of a polymer directly in course of preliminary irradiation of the system at $77 \mathrm{~K}$. The polymer softens a solid matrix of reagents and impedes brittle destruction of a sample. A key role of brittle destruction in the autowave cryo-chemical conversion mechanism was established in [1], [2]. It was reported in [13] that the occurrence of plastic deformation stress is not enough to initiate cryo-chemical conversion in mode of travelling waves. At low radiation doses a small amount of polymer formed at $77 \mathrm{~K}$ does not affect the process. A portion of polymer increases with radiation dose, the sample becomes less brittle and more plastic. As a result, the conversion wave propagation decelerates not only if the reaction is initiated in bottom of the sample ( $>60 \mathrm{kGy}$ dose, Figure 1, curve 2 ) but if it is initiated in top part of the sample ( $>100 \mathrm{kGy}$ dose, Figure 1, curve 1). This factor affects both for detonation and thermal mechanisms of travelling wave cryo-processes in solid reagent matrices, at that such effect being stronger for the detonation mechanism than for thermal one.

The possibility of the formation of acetaldehyde polymers at $77 \mathrm{~K}$ under $\gamma$-radiation and also under photolysis was shown in [6], [14]. The accumulation of polymer under $\gamma$-radiation at $77 \mathrm{~K}$ was observed for co-polymerization of HCN and ethylene oxide [9]. The formation of copolymer is almost a linear process with a radiation-chemical yield equal to 20 molecules per $100 \mathrm{eV}$ of absorbed energy.

The following experiment was performed to prove the hypothesis. Propagation velocities were compared for two samples irradiated by equal $\gamma$-radiation dose. A certain additional amount of polymer was added to one of the samples before irradiation. If deceleration of conversion velocity is due to a plasticizing effect of a polymer formed in course of irradiation, an additional homogeneous insertion of a polymer into a sample should provide a lower travelling wave propagation velocity as compared with a polymer-free sample.

Because of impeded homogeneous distribution of additionally inserted polymer along an extended cylindrical sample and to maximally approximate the properties of inserted polymer to those of a co-polymer produced under irradiation at $77 \mathrm{~K}$, the experiment was performed as follows. The sample was $\gamma$-irradiated at $77 \mathrm{~K}$ by a small dose $1 \mathrm{kGy}$. Then it was slowly heated up to 142-145 K (m.p. = $140 \mathrm{~K}$ ). According to the data on thermally activated post-polymerization [12], heating is accompanied by the formation of $4.0 \%$ of co-polymer. Then the sample was cooled down to $77 \mathrm{~K}$ and irradiated by a $55 \mathrm{k}$ Gy dose. At $77 \mathrm{~K}$ a local brittle destruction was organized in the bottom of the prepared sample. In accordance with the data presented in Figure 1 (curve 2) for the sample irradiated by a similar dose (without inserting additional polymer), local brittle destruction in bottom of the sample provides the appearance of a travelling conversion wave whose velocity is $1870 \mathrm{~mm} / \mathrm{s}$. When local brittle destruction was realized in bottom of the sample containing additional co-polymer, it provided the appearance of a travelling conversion wave whose velocity was essentially lower - $130 \mathrm{~mm} / \mathrm{s}$ (see a triangular point in Figure 1). This value is near to that of wave propagation initiated in top of the sample. The temperature profile of the travelling wave is shown in Figure 2b, curve 3.

Thus, high-velocity (detonation-like) propagation of travelling co-polymerization wave is realized in a glassy HCN solution in acetaldehyde. The initiation of co-polymerization of acetaldehyde 
and $\mathrm{HCN}$ by a local brittle destruction in the bottom of a sample at $77 \mathrm{~K}$ allows more complete use of mechanical energy accumulated in solid matrix of reagents to initiate travelling conversion waves (non-thermal shock-wave, detonation-like mechanism). The wave front propagation velocity attains $1870 \mathrm{~mm} / \mathrm{s}$ for a $55 \mathrm{kGy}$ dose. Nevertheless, a co-polymer formed under pre-irradiation at $77 \mathrm{~K}$ plasticizes a solid matrix of reagents. It is a reason for degeneration of high-velocity co-polymerization wave propagation modes at high radiation doses. The co-polymerization wave propagation velocities decrease to $50 \mathrm{~mm} / \mathrm{s}$ at a $200 \mathrm{kGy}$ dose. Similar propagation velocities are observed if reaction is initiated by brittle destruction in top of the sample.

In the framework of the model considered in this work, the softening of the solid matrix observed for high doses of radiation, can be taken into account by means of the Young modulus (parameter $b$ in the dimensionless model). Its decrease results in the decrease of the wave speed.

\section{Conclusions}

The mechanism of propagation of cryo-chemical waves discussed in this work can be used for the description of some dynamical phenomena in related fields of solid phase physics. In particular, this concerns geotectonic phenomena and earthquakes in relation with gasless detonation and fracture of solids and with the supersonic wave theory created in [18], [19] for the explanation of catastrophically fast decays of metastable phase states in solids. This unconventional approach which takes into account fracture mechanics was also developed for different solid phase transformations in physics of semiconductors and in physics of explosives from the point of view of their sensibility to friction and shock.

The possibility of application of this theory to geophysical processes is based on the hypothesis that geotectonic phenomena and earthquakes result from catastrophic phase transitions in rocks of the Earth crust. The classical theory of polymorphism describes metastable phases in rocks but it does not investigate dynamics of transitions between them. The works cited above develop some methods of bifurcation theory to study them.

These phenomena are modelled by coupling reaction-diffusion equations describing chemical or phase transformations, and the wave equation, describing elastic perturbations. The feedback coupling considered in this work involves (i) a dependence of the sound velocity on the phase (chemical) field, and (ii) the destruction (fracture) of initial phase equilibrium when the stress exceeds a critical value: such critical stress destroys the matrix because of the difference in the initial and final product densities and induces phase transformation. An exhaustive analytic and numerical study of travelling waves of fracture reveals the existence of supersonic modes of transformations. An important problem for geotectonics of initiation of earthquake catastrophic waves by local disturbance in the Earth rocks is investigated. The present model allows the determination of the critical stress necessary to ignite gasless detonation by local perturbations.

The concept of phase transformation was used for the explanation of earthquake phenomena in [15], [23]. The question about a possible laboratory experiment on modelling of earthquakes is discussed with the example of super-fast decay of metastable silicate glasses - chemical analogues of the rock material (explosions of "Prince Ruppert Drops" [17] and of "Tempered Glasses" [16]). 


\section{Acknowledgements}

The authors are grateful to L.S.Kiryukhina who performed elemental analysis of copolymers. The work is performed with the financial support of RFBR grant No. 07-03-00259

\section{References}

[1] V.V. Barelko, I.M. Barkalov, V.I. Goldanskii, D.P. Kiryukhin, A.M. Zanin. Autowave modes of conversion in low-temperature chemical reactions in solids. Advances in Chem. Phys. 74 (1988), 339-384.

[2] V.V. Barelko, I.M. Barkalov, V.I. Goldanskii, A.M. Zanin, D.P. Kiryukhin. High-velocity autowave regimes of transformations in low-temperature chemistry of solids. Usp. Khim., 59 (1990), No. 3, 353-374.

[3] V.V. Barelko, D.P. Kiryukhin, A.M. Zanin, I.M. Barkalov. Is it probable the idea about unique role of stabilized radicals and ions in the kinetic of radiation chemical solid state transformation? Khim. Vys. Energ., 24 (1990), No. 2, 103-107.

[4] G.A. Kichigina, D.P. Kiryukhin, A.M. Zanin, I.M. Barkalov. Auto-wave regime of lowtemperature radiation-induced polymerization of cyclopentadiene. Vysokomol. Soedin., Ser.A, 24 (1990), No. 5, 1094-1099.

[5] D.P. Kiryukhin, V.V. Barelko, I.M. Barkalov. Travelling waves of cryochemical reactions in radiolyzed systems. Khim. Vys. Energ., 33 (1999), No. 3, 133-144.

[6] D.P. Kiryukhin, I.M. Barkalov. Radiative post polymerization of solid acetaldehyde. Vysokomol. Soedin., Ser. A, 21 (1978), No. 5, 1021-1025.

[7] D.P. Kiryukhin, I.M. Barkalov. The autowave regimes of cryopolymerization. Vysokomol. Soedin., Ser.B, 42 (2000), No. 9, 1604-1615.

[8] D.P. Kiryukhin, I.M. Barkalov. Chain chemical reactions at low temperatures. Usp. Khim., 72 (2003), No. 3, 245-261.

[9] D.P. Kiryukhin, G.A. Kichigina, I.M. Barkalov. Low-temperature radiation copolymerization of hydrogen cyanide with ethylene oxide. Vysokomol. Soedin., Ser. B, 40 (1998), No. 6, 1044-1047.

[10] D.P. Kiryukhin, G.A. Kichigina, P.S. Mozhaev, I.M. Barkalov. Cryopolymerization of hydrogen cyanide with acetaldehyde. Vysokomol. Soedin., Ser. A, 39 (1997), No.7, 1109-1114.

[11] D.P. Kiryukhin, G.A. Kichigina, P.S. Mozhaev, I.M. Barkalov. Autowave regime of cryopolymerization of acetaldehyde with hydrogen cyanide. Vysokomol. Soedin., Ser. A, 40 (1998), No. 2, 236-240. 
[12] D.P. Kiryukhin, P.S. Mozhaev, V.V. Barelko. About detonation mechanism of autowave phenomena in cryochemical solid phase processes. Khim. Fiz., 11 (1992), No. 2, 264-268.

[13] D.P. Kiryukhin, A.M. Zanin, V.V. Barelko, I.M. Barkalov. The role of loading and fracture of sample in initiation of autowave regimes of solid phase cryochemical transformations. Khim. Fiz., 5 (1986), No. 8, 1130-1134.

[14] E.S. Mansueto, C.A. Wigth. Photopolymerization of disordered solid acetaldehyde at cryogenic temperatures. J. Phys. Chem., 96 (1992), 1502-1054.

[15] C. Meade, R. Jeanloz. Acoustic emissions and shear instabilities during phase transformations in Si and Ge at ultrahigh pressures. Nature, 339 (1989), 616-618.

[16] J.S. Olcott. Chemical strengthening of glass. Science, 140 (1963), 1189.

[17] T. Polyakova, I. Fomin, D. Karpinskii. Features of destruction of Batav (Prince Ruppert) glass tears. Doklady Akademii Nauk, 311 (1993), 431-433.

[18] A. Pumir, V. Barelko. Detonation type waves in phase (chemical) transformation processes in condensed matter. Eur. Phys. J. B, 10 (1999), 379-383.

[19] A. Pumir, V. Barelko. Propagation and ignition of fast gasless detonation waves of phase or chemical transformation in condensed matter. Eur. Phys. J. B, 16 (2000), 137-145.

[20] A. Pumir, V. Barelko. Could ignition of combustion-like waves of cryo-chemical reactions in solids. Eur. Phys. J. B, 22 (2001), No. 1, 71-77.

[21] F.M. Rapoport, A.A. Il'inskaya. Laboratory methods for preparation of pure gases. Goskhimizdat, Moscow, 1963.

[22] G. S. Sih. On the singular character of thermal stresses near a crack tip. J. Applied Mechanics, 29 (1962), Trans. ASME, Vol. 84, Series E, 587-589.

[23] D. Sornette. Earthquakes: from chemical alteration to mechanical rupture. Physics Reports, 313 (1999), No. 5, 238-292. 